\title{
Automatic Measurement Device for Product Components
}

\author{
Hong-Jun GUa , Shi-Dong ZHU, Ding-Zhu XUE , Jian LI, Li-Xin HOU, \\ Shi-Jun LI ${ }^{\mathrm{b},{ }^{*}}$ \\ ${ }^{1}$ Jilin Agricultural University, Changchun 130118, Jilin, China \\ aghj_dennis@163.com, ${ }^{b}$ Isj0883@sina.com \\ *Shi-Jun LI
}

Keywords: automatic measurement, SCM, moving component, infrared sensor.

\begin{abstract}
An automatic measurement device is designed to measure the length of product components on the conveyor in the factory, and count them. This system uses single chip microcomputer (SCM) STC89C52 as the core, which can control driver circuit to run the stepping motor driving the conveyor. The moving component length can be calculated by the rotating speed of stepping motor and the interval of infrared sensor blocked. The displaying screen LCD1602 can show the length data and counts, and voice chip ISD4003 can broadcast them. Software adopts C language with readability and good portability to write the program.
\end{abstract}

\section{Introduction}

In the process of production, the user is strict with the the accuracy of the component length. The workers measure the samples by vernier caliper, which results in some measuring error and can not ensure the quality of every component. For the phenomenon, an automatic measuring device can be introduced to improve the measurement accuracy, reduce the manpower and increase the production efficiency[1].

Automation and intelligence in the industry have been widely used with the rapid development of science and technology[2]. Sensor plays an important role in the automatic examining and controlling system[3]. The infrared sensor detection is non-contact, which has many advantages, such as, eliminating the interference of contact detection on flexible objects, measuring some objects with radiation and high temperature, and high acquiring information speed and so on. So this design uses infrared sensor E3F-DS30P1 as the detection element[4].

SCM has many virtues, such as, small volume, low power consumption, strong control function, miniaturization, flexible extension, good integration and convenient use, etc. And SCM often appears in the fields of automatic examining and controlling[5].

\section{System Hardware Design}

\section{Overall Design Summary}

This system presented in this paper includes five main parts: displaying screen, voice broadcasting, infrared sensor, stepping motor driver and SCM. The overall design diagram is shown in Fig .1.

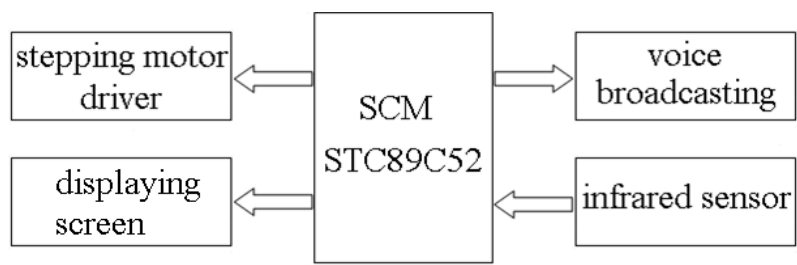

Fig. 1 The overall design diagram

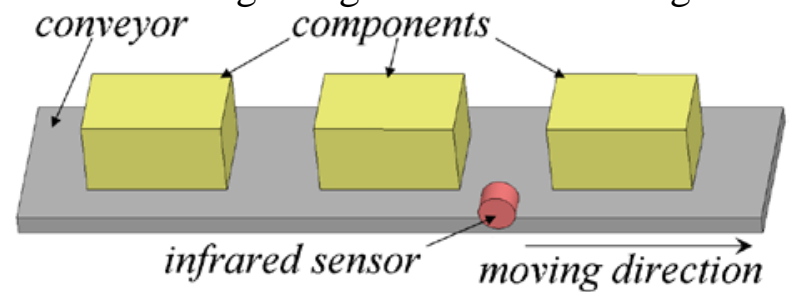

Fig. 2 The detecting diagram

SCM adopts STC89C52 as the control core which sends pulses to stepping motor driver. Then the motors runs conveyor on which the components follow. Infrared sensor receives the light sent by itself when the component move to the front of the sensor, and at the moment SCM starts the timer. 
When infrared sensor can not receive the light, SCM stops the timer. According to the time and the speed of the motor, the length of one component can be calculated. The number of components and cumulative lengths can be got. The detecting diagram is shown in Fig. 2.

\section{Stepping motor driver}

As shown in Fig. 3, stepping motor diver mainly consists of L297 and L298. The ports P1.0, P1.1 and P1.2 of SCM are connected with CW/ $\overline{\mathrm{CCW}}$, ENABLE and $\overline{\mathrm{CLOCK}}$ of L297, respectively. The pin CW/ $\overline{\mathrm{CCW}}$ decides the forward/backward, ENABLE decides whether L297 works, and $\overline{\text { CLOCK }}$ receives the pulses from SCM. The ports OUTPUT1, OUTPUT2, OUTPUT3, OUTPUT4 of L298 are connected with the motor. The speed of motor depends on the pulse frequency.

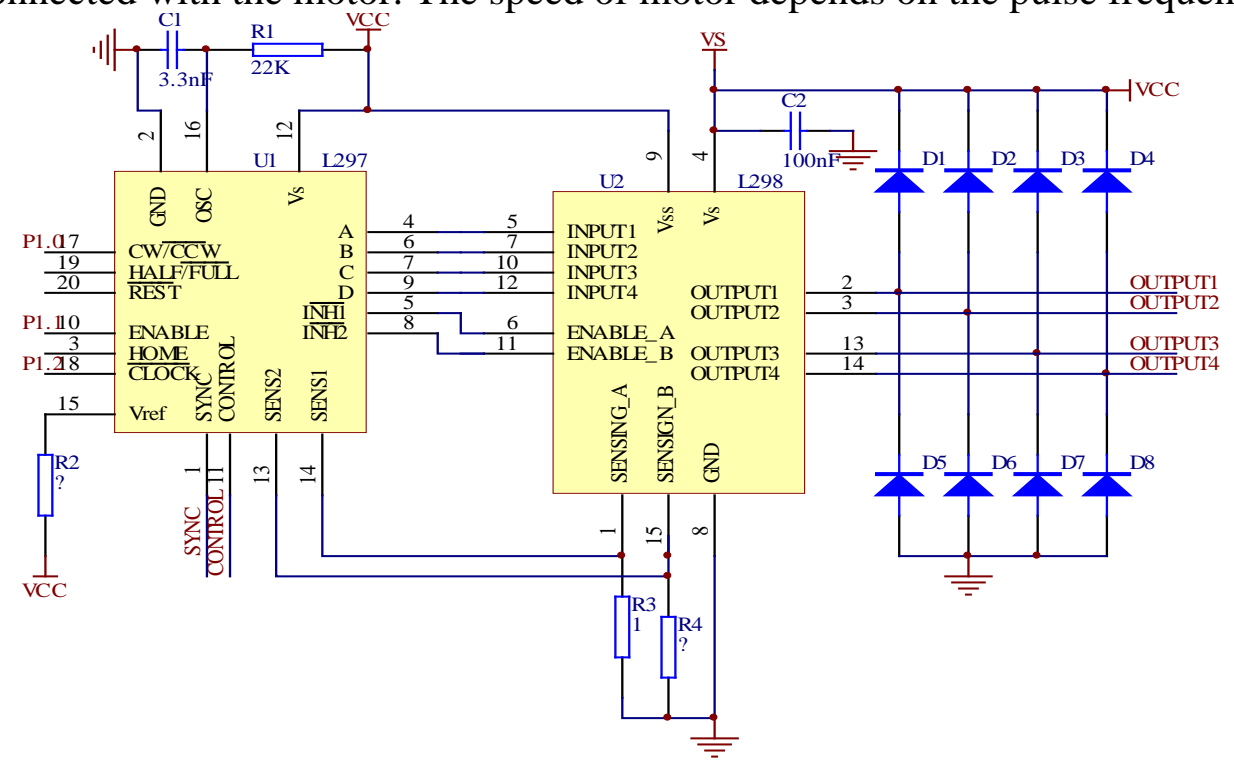

Fig. 3 The stepping motor driver diagram

\section{System displaying screen}

The circuit of the displaying screen LCD1602 connected with SCM is shown in Fig. 4. The bidirectional parallel ports, DB0 to DB7, are connected with the port P0 of SCM. The ports can send contents from SCM to LCD1602. R/S port decides the contents are data or commands. R/W port decides read or write operation. E port can receive the pulses from SCM, then the data and commands can be input in the LCD1602.

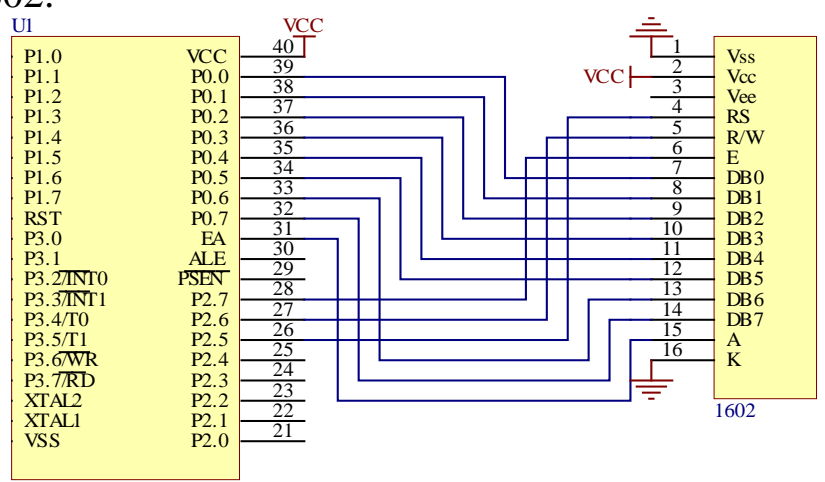

Fig. 4 The system displaying screen diagram

\section{Voice broadcasting module}

Voice broadcasting module design diagram is shown in Fig. 5. Voice recording-playing module adopts ISD4003 with SPI bus. The connection between SCM and ISD4003 is simple. The output voice signals of ISD4003 are amplified by LM386. Analog voice signals can be input by Mac and stored in the ISD4003. 


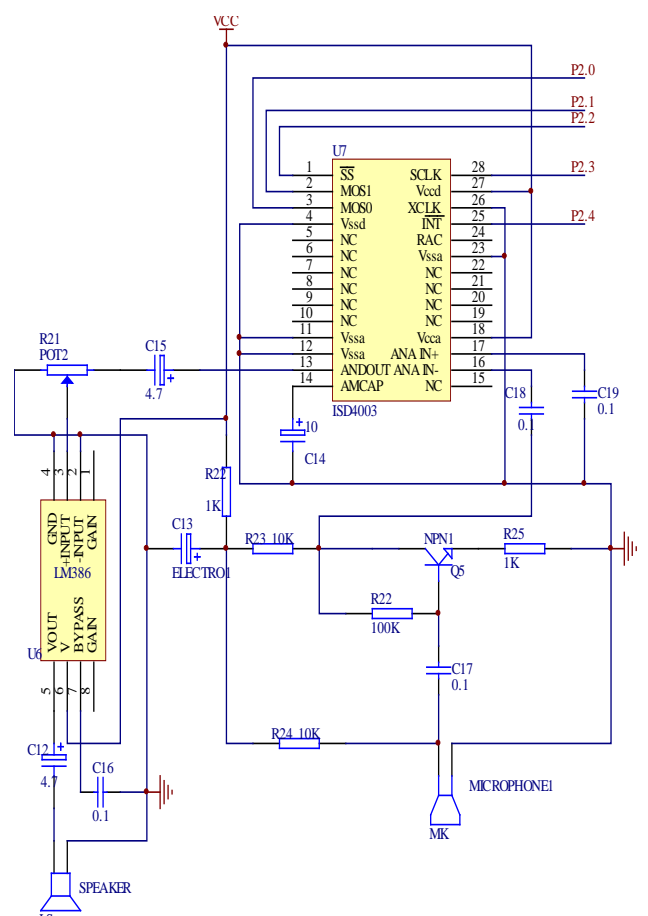

Fig. 5 The Voice broadcasting module

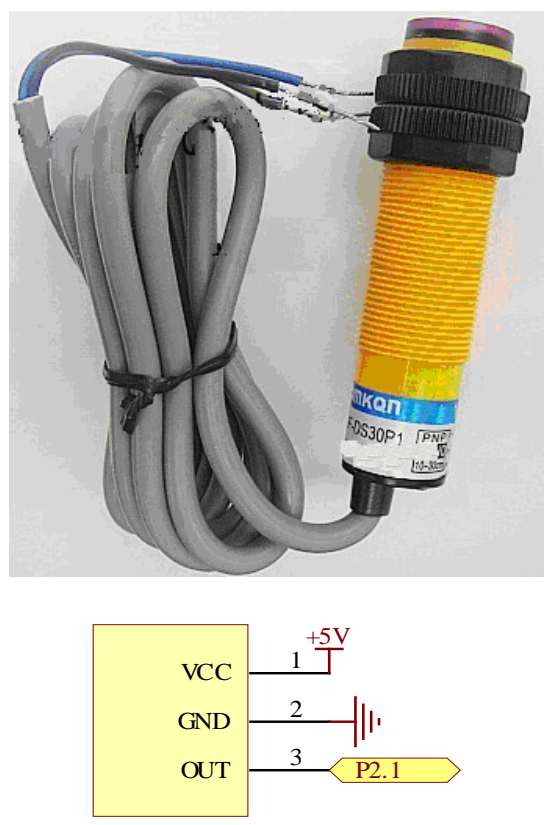

Fig. 6 The system displayer diagram

\section{Infrared sensor detection module}

In Fig. 6, the above chart gives the infrared sensor E3F-DS30P1, and the below one gives the pins of the sensor. E3F-DS30P1 is a transmission-receiving module and has some remarkable features, for instance, stable performance, fast respond speed, impact and vibration resistant, small light interference and long life, etc. This sensor with three wire system is easy to connect with other electronic components. And it can detect many different objects, such as, glass, iron, plastic, wood, liquid and so on, so it applies to the mechanical, chemical, paper making, light industry and other aspects. E3F-DS30P1 has three pins, and the output pin connects the port P2.1 of STC89C52. When P2.1 is ' 0 ', it means something shields the sensor.

\section{System Software Design}

\section{Stepping motor running program}

Stepping motor driver gets pulses from STC89C52 to run the motor. When the user presses the 'run' key, STC89C52 starts the timer to generates pulses, then the motors rotate. When the 'stop' key is pressed, STC89C52 halts the timer to stop the motor. The corresponding software flow diagram is shown in Fig. 7(a).

\section{Voice broadcasting program}

Fig. 7(b) shows the software flow diagram of voice broadcasting program. When the key 'play' is pressed, ISD4003 can broadcast the length of current component, the length sum and count of components.

\section{Displaying program of LCD1602}

As shown in Fig. 7(c), the displaying screen can show the information which ISD4003 broadcasts. Firstly, LCD1602 need the initialization including the following processes: set 8-bit displaying format, open or close cursor and set cursor flash, set input mode with right shift and clear the full screen, etc. Secondly, judge whether the displaying screen is busy. Lastly, if the displaying screen is not busy, it can show the related component information. According to the rotation direction of motors, the displaying device can indicate show the moving direction of components. 


\section{Infrared sensor detecting program}

In the infrared sensor, the infrared emitter works all along, and the infrared receiver can get the reflected light when the component shields the emitter. When the top of the component shields the the emitter, the timer is started. When the component bottom can not shields the emitter, the timer stops. The component length can be calculated according to the time and the motor speed. The process shown Fig. 7(d).

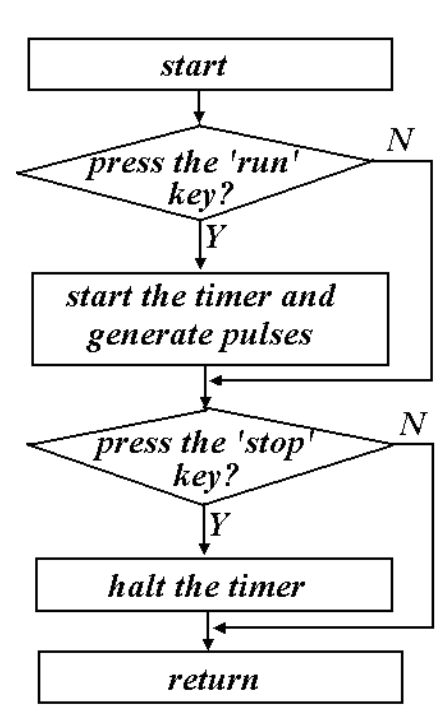

(a)

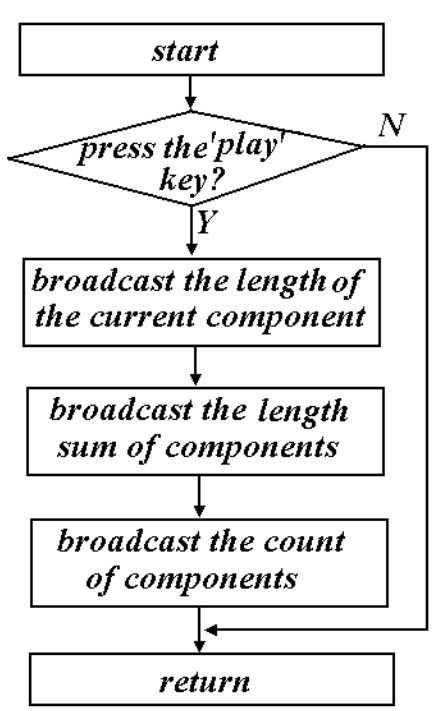

(b)

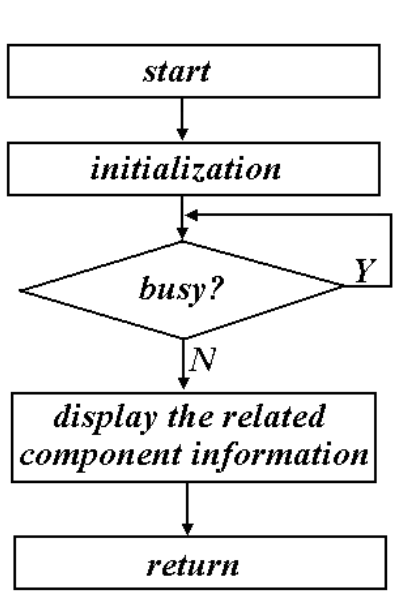

(c)

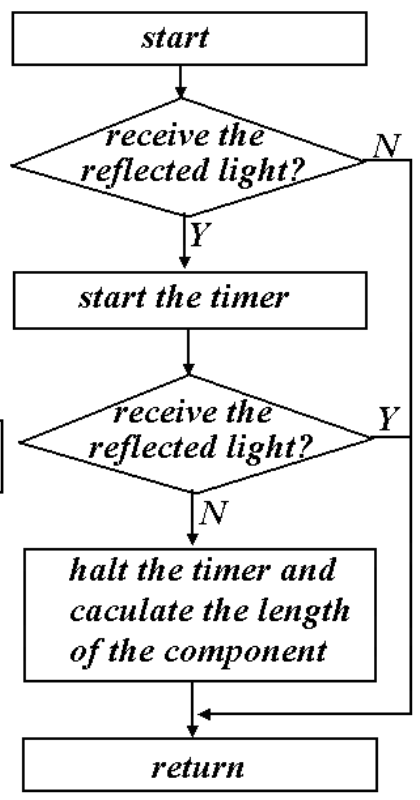

(d)

Fig. 7 System software flow diagram

\section{Conclusions}

This paper presents an automatic measurement device measuring the length of product components on the conveyor in the factory. By testing some times, this system works stability, has high accuracy, helps to improve the efficiency, and has guiding significance to the detecting work in the factory in the future.

\section{Acknowledgments}

The corresponding author, Shi-Jun LI, and other authors wish to express their gratitude to the projects, Jilin Province University Students' Innovation and Entrepreneurship Project, Jilin Province Economic Structural Adjustment Leading Fund Special Project (No. 2014Y108) and Changchun City Science and Technology Plan Project (No. 14nk029), Jilin Agricultural University, for their generous support of this work.

\section{References}

[1] Hong Zhou, Xiaotian Lu, Chengfei He: Computer Knowledge and Technology. Vol. 11(2015) p.265-268, in Chinese.

[2] Liguo Wang: Manufacturing Automation. Vol. 33 (2011) p.20-22, in Chinese.

[3] Wen Zhang: Techniques of Automation \& Applications. Vol. 32 (2013) p.99-101, in Chinese.

[4] Zhengwu Yao: Manufacturing Automation. Vol. 36 (2014) p.125-130, in Chinese.

[5] Lei Lu, Zurong Wang, Xia Mei: Journal of Yangtze University. Vol. 8 (2011) p.110-117,127, in Chinese. 